\title{
DIRECT MEASUREMENTS OF THE DIRECTIVITY OF TYPE I AND TYPE III RADIATION AT $169 \mathrm{MHz}$
}

\author{
C. CAROUBALOS and J. L. STEINBERG \\ Observatoire de Meudon, France
}

\begin{abstract}
A $169 \mathrm{MHz}$ radiometer fed from a Sun-pointed $8 \mathrm{~dB}$ antenna was flown on the Soviet probe Mars-3 in 1971-1972. This experiment called Stereo-1 is part of a cooperation program between the U.S.S.R. and France.

Analysis of the data shows that the spacecraft systems and the experiment performed satisfactorily throughout the mission and that absolute calibrations on the Earth and on board the spacecraft are reliable. The stereo angle $\theta$ between the observing directions varied from 0 to $76^{\circ} ; 185 \mathrm{~h}$ of observations have been logged including several hundreds of type III's and about 10000 type I bursts which have not yet been analyzed in detail.
\end{abstract}

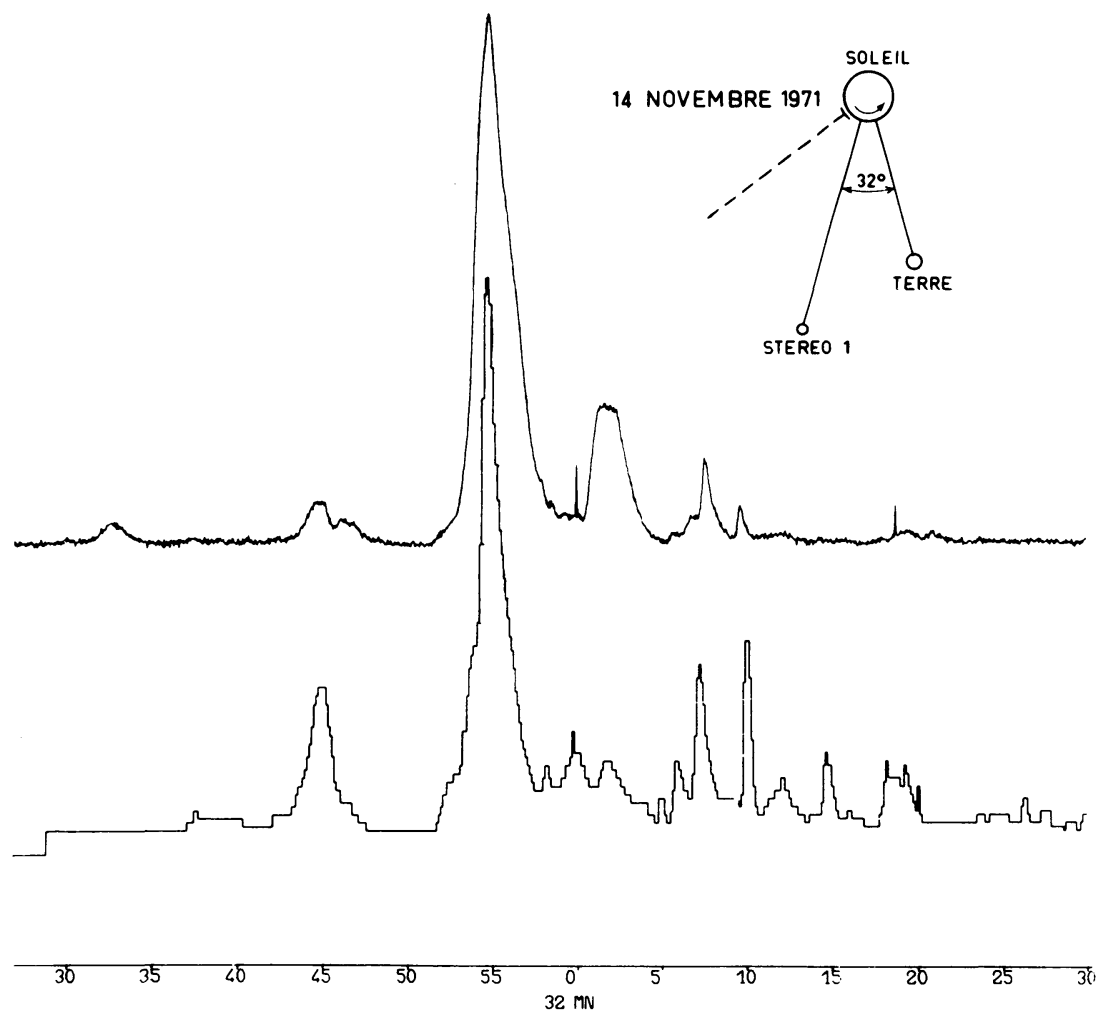

Fig. 1. A group of $169 \mathrm{MHz}$ type III bursts recorded on the ground (top) and on board the Mars-3 space probe. Stereo angle was $33^{\circ}$. The dotted line gives the radial direction through the active region where those events took place. 
The first results of a partial analysis of type I and type III bursts are:

\section{Type III}

- The time profile of isolated type III's at $169 \mathrm{MHz}$ is independent of the observing direction; for instance, there are only negligible propagation effects on the decay time of these events.

- This property has been used to separate individual events when they overlap; it has thus been possible to identify fundamental and harmonic pairs among the November 14, 1971 events which took place at about $70^{\circ} \mathrm{E}$ longitude as seen from the Earth. $80 \%$ of these events are harmonics without detectable fundamentals.

- The directivity ratio $R$ is defined as the ratio of the event intensity in the probe direction to its intensity in the Earth direction. $R$ is constant over individual events but can change from that event to the next. The distribution of $R$ 's over a daily observing period (shorter than $1 \mathrm{~h}$ ) can change from day to day between two extremes:

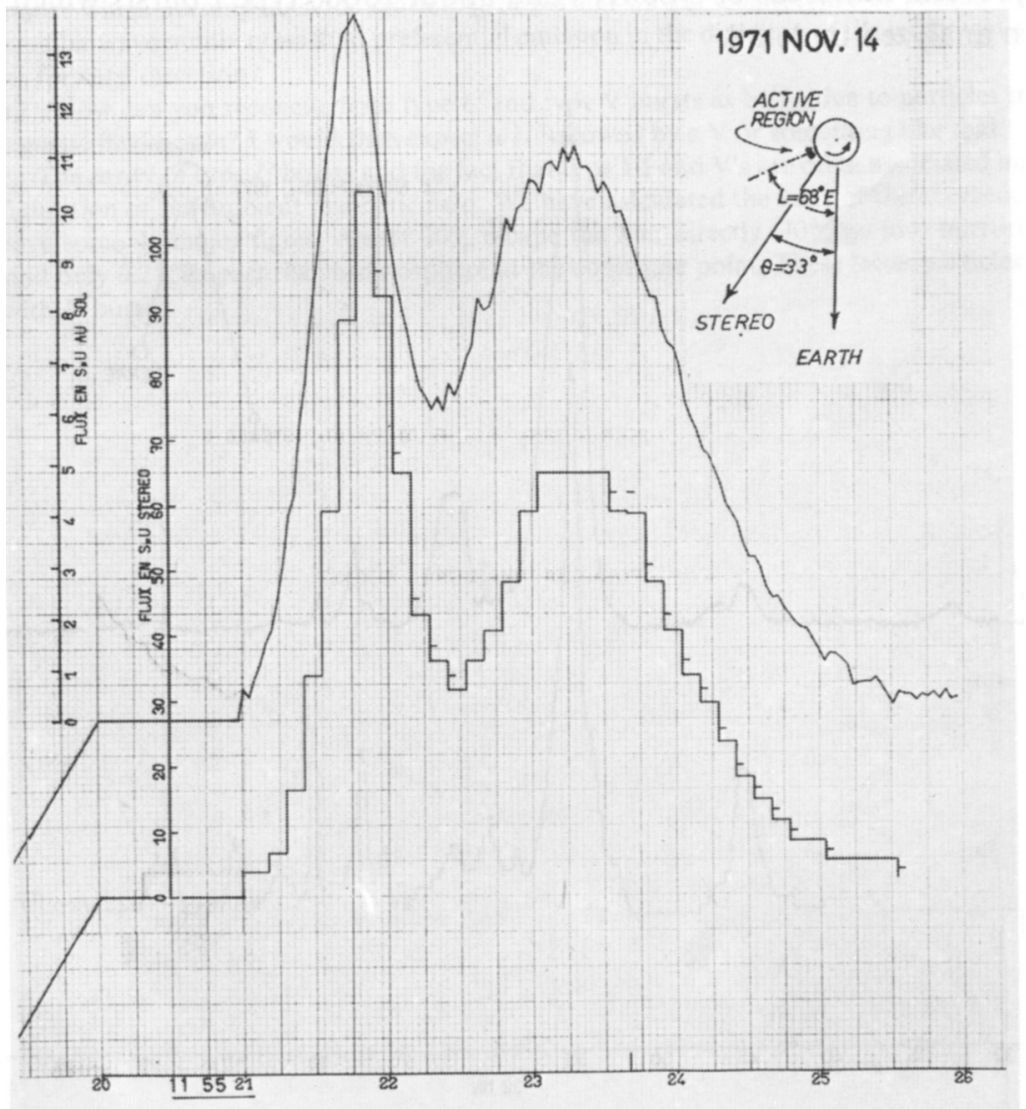

Fig. 2. A typical fundamental-harmonic pair from the 6 pairs observed on November 14, 1971. The fundamental component is always found more directive than the harmonic. 
- The range of $R$ 's can be narrow around some stable mean value which depends upon the geometrical configuration (source position, lines of sight); in these cases, the source is seen close to the Sun's center from both observing stations.

- The $R$ values are widely scattered around a mean value which can be time dependent; in most of these cases, the source is close to the limb. On November 14, 1971, fundamental components are more directive than their associated harmonic components $\left(\theta=33^{\circ}\right)$ and $R$ ranges from 1 to 50 .

- The observed directivity is always higher than predicted from spherically symmetric coronal models where the scattering power is large enough to account for a noticeable part of the type III sizes and for the relative positions of the fundamental and harmonic components. For sources near the center, the difference between the computed and observed $R$ 's can be made small only if the scattering power is small and the harmonic source directive; moderate angular shifts of the computed radiation pattern are generally required. For limb sources large deviations from spherical symmetry have to be built into the models.

- It has been shown that streamer models in an otherwise spherical corona cannot fit the data better than spherical models.

\section{Type I}

Type I radiation is strongly beamed in space; the correlation between Mars-3 and
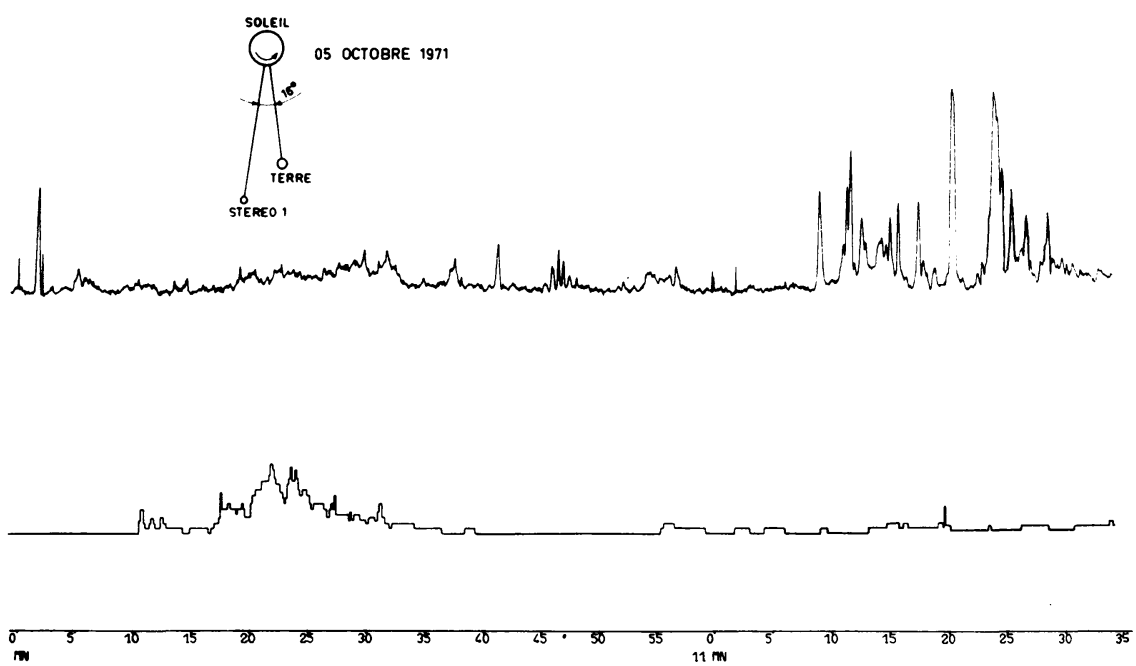

Fig. 3. Groups of $169 \mathrm{MHz}$ type I bursts recorded on the ground (top) and on board the Mars-3 space probe. Decorrelation is evident with a stereo-angle of only $16^{\circ}$.

Earth records drops to zero for a stereo angle of $\sim 20^{\circ}$. However in some cases a good correlation is observed for larger angles (i.e. $27^{\circ}$ or even $35^{\circ}$ ).

The present preliminary report is based on an extensive analysis of only part of the available data. 
Notes added in proof. On Figure 1 the Stereo angle should read $33^{\circ}$.

The material summarized above is now published in full in:

Caroubalos, C. and Steinberg, J. L.: 1974, Astron. Astrophys. 32, 245.

Caroubalos, C., Poquerusse, M., and Steinberg, J. L.: 1974, Astron. Astrophys. 32, 255. 